\title{
One-step synthesis of a mid-chain functional macrophotoinitiator of a polystyrene-poly( $\varepsilon$-caprolactone) diblock copolymer via simultaneous ATRP and ROP using a dual-functional photoinitiator
}

\author{
Mustafa Degirmenci, Cafer Gokkaya and Mustafa Durgun
}

A one-step synthesis of a well-defined mid-chain functional macrophotoinitiator of a polystyrene-poly( $\varepsilon$-caprolactone) diblock copolymer (PSt-PI-PCL) was successfully performed at $110^{\circ} \mathrm{C}$ using a novel dual-functional photoinitiator via simultaneous atom transfer radical polymerization and ring-opening polymerization (ROP). This dual-functional photoinitiator (Br-PI-OH), possessing a bromine group on one end and a hydroxyl group on the other, was synthesized by the reaction of the photoinitiator 2-hydroxy4'-(2-hydroxyethoxy)-2-methylpropiophenone (HO-PI-OH) with 2-bromopropanoyl bromide. The two distinct polymerization reactions initiated by $\mathrm{Br}-\mathrm{PI}-\mathrm{OH}$ proceeded in a controlled manner without mutual interference. Elemental analysis and ${ }^{1} \mathrm{H}-\mathrm{NMR}$, ${ }^{13} \mathrm{C}$-NMR and Fourier transform infrared spectroscopy (FT-IR) spectroscopy were used to characterize the chemical structure of Br-PI-OH. Characterization of the macrophotoinitiator PSt-PI-PCL was achieved using ${ }^{1} \mathrm{H}-\mathrm{NMR}$, FT-IR, gel permeation chromatography, UV-vis and fluorescence spectroscopy.

Polymer Journal (2016) 48, 139-145; doi:10.1038/pj.2015.93; published online 4 November 2015

\section{INTRODUCTION}

Structurally well-defined block copolymers are an important class of polymeric materials owing to their technological applications and extraordinary properties. Preparation of such block copolymers is generally achieved through sequential addition of monomers to a living polymerization, controlled/living polymerization systems or the transformation approach. ${ }^{1}$ Among these, the use of a dual or heterofunctional initiator to combine two distinct controlled/living polymerization methods is the preferred technique because of the possibility of a one-step process and its applicability to a wide range of monomers. ${ }^{2-5}$ Dual initiators contain two functional groups that can initiate two mechanistically different polymerizations independently without any intermediate transformation steps. Recently, one-step processes using dual initiators successfully synthesized well-defined block copolymers through several combinations of polymerization techniques, such as nitroxide-mediated polymerization with ringopening polymerization (ROP), ${ }^{6,7}$ atom transfer radical polymerization (ATRP) with nitroxide-mediated polymerization, ${ }^{6}$ reversible addition-fragmentation chain transfer polymerization with ROP, ${ }^{5,8-10}$ as well as ATRP with ROP. ${ }^{4,11}$

Few studies have investigated the synthesis of well-defined block copolymers in a one-step process that combines ATRP with ROP. However, such a process was used by Mecerreyes et al. ${ }^{6}$ to synthesize PMMA- $b$-PCL, using 2,2,2-tribromoethanol as the dual initiator; Nasser-Eddine et $a l .{ }^{4}$ to prepare PtMMA- $b$-PCL; and more recently,
Kryuchkov and co-workers ${ }^{11}$ to synthesize amphiphilic diblock copolymers of lactide and 2-dimethylaminoethyl methacrylate in a one-pot approach. Using an approach that is distinct from those in the literature, in this study, we synthesized a polystyrene-poly( $\varepsilon$-caprolactone) diblock copolymer (PSt-PI-PCL) via simultaneous ATRP and ROP using a dual-functional photoinitiator in a one-step process. The resultant block copolymer contains a photofunctional group in the center of the polymer chain, and this polymer is known as a midchain functional macrophotoinitiator.

In recent years, there has been marked interest in the synthesis of macrophotoinitiators as they possess a combination of the properties of polymers and low molecular weight photoinitiators. ${ }^{12-15}$ The advantages expected from macrophotoinitiators in ultraviolet (UV) curing applications include good compatibility, high reactivity, low migration and low volatility, which should reduce the odor problems associated with low molar mass photoinitiators. ${ }^{16,17}$ Macrophotoinitiators are also used as precursors for block and graft copolymers. ${ }^{18-20}$ Two methods have been proposed for the preparation of well-defined macrophotoinitiators: (i) the introduction of photoactive groups into the polymer using photofunctional compounds as initiators in a controlled/living polymerization, such as ATRP or ROP; (ii) the functionalization of the final polymer prepared by these techniques (ATRP or ROP) using initiators with suitable functional and photoreactive groups. ${ }^{21-23}$ Many studies have aimed to synthesize well-defined end- or mid-chain functional macrophotoinitiators of 
polystyrene (PSt), ${ }^{23-26}$ poly(methyl methacrylate) (PMMA) ${ }^{27}$ and/or poly $\left(\varepsilon\right.$-caprolactone) $(\mathrm{PCL})^{28-30}$ using the above methods.

We have previously synthesized well-defined macrophotoinitiators with mid- or end-chain photofunctional groups from brominefunctionalized photoinitiators with ATRP25,27 and hydroxylfunctionalized photoinitiators with ROP, ${ }^{28,29}$ as well as by functionalization of polymers obtained from ATRP or ROP using initiators with suitable functional and photoreactive groups. ${ }^{20,22,26,30}$ All of the macrophotoinitiators that we prepared using these methods were homopolymers. As part of our continuing interest in the synthesis (by various methods) and characterization of novel polymers possessing a photofunctional group in the middle or at the end of their chains, in this study, we combined ATRP and ROP in a one-step strategy. Many studies related to homopolymer-based macrophotoinitiators can be found in the literature, but there are no studies on the synthesis and characterization of copolymer-based macrophotoinitiators. To the best of our knowledge, in this study we provide the first reported synthesis of a well-defined block-type mid-chain macrophotoinitiator of PSt-PIPCL via a combination of ATRP and ROP in a one-step process using a dual-functional photoinitiator containing a bromine group on one end and a hydroxyl group on the other.

\section{EXPERIMENTAL PROCEDURE}

\section{Materials}

$\varepsilon$-Caprolactone ( $\varepsilon$-CL) (Sigma-Aldrich, Munich, Germany), styrene (St) (Sigma-Aldrich) and methyl methacrylate (MMA) (Sigma-Aldrich) were dried over $\mathrm{CaH}_{2}$ followed by distillation under reduced pressure. The photoinitiator 2-hydroxy-4'-(2-hydroxyethoxy)-2-methylpropiophenone (HO-PI-OH, also known as Irgacure 2959) was purchased from Sigma-Aldrich and used without further purification. 2-Bromopropanoyl bromide (Sigma-Aldrich), $\mathrm{CuBr}$ (Sigma-Aldrich), 2,2'-bipyridine (Merck, Kenilworth, NJ, USA), pyridine (Lab-scan, Gliwice, Poland) and stannous 2-ethylhexanoate $\left(\mathrm{Sn}(\mathrm{Oct})_{2}\right)$ (Sigma-Aldrich) were also used as received. Dichloromethane $\left(\mathrm{CH}_{2} \mathrm{Cl}_{2}\right)(\mathrm{Lab}-$ scan) and tetrahydrofuran (THF) (Sigma-Aldrich) were dried and purified by previously reported methods. Methanol (Lab-scan) and all other chemicals were used as received.

\section{Synthesis of the dual-functional photoinitiator (Br-PI-OH)}

HO-PI-OH (1.12 g, $5 \mathrm{mmol})$, dry THF $(30 \mathrm{ml})$ and pyridine $(0.72 \mathrm{~g}, 10 \mathrm{mmol})$ were combined in a $250-\mathrm{ml}$ three-necked round-bottom flask fitted with a condenser, a magnetic stirrer bead, a nitrogen inlet-outlet and an addition funnel containing 2-bromopropanoyl bromide (1.08 g, $5 \mathrm{mmol}$ ) and $30 \mathrm{ml}$ of a dry THF mixture. The flask was placed in an ice-water bath. The solution of 2-bromopropanoyl bromide was added dropwise over a period of $45 \mathrm{~min}$ under nitrogen. Then, the mixture was allowed to reach room temperature and was stirred for $20 \mathrm{~h}$. The resultant white precipitate was removed by filtration, and the solution was washed three times with $1 \mathrm{M} \mathrm{HCl}$ and twice with water. Then, the solution was dried with $\mathrm{MgSO}_{4}$, and the solvent was removed by vacuum distillation. Finally, the remaining product was purified on a silica column and eluted with $\mathrm{CH}_{2} \mathrm{Cl}_{2} / \mathrm{THF}$ (3:1) to yield $\mathrm{Br}-\mathrm{PI}-\mathrm{OH}$ as a viscous orange-yellow liquid (yield: $1.47 \mathrm{~g}, 82 \%$, after evaporation of the solvent). All steps were performed in the dark.

$\mathrm{C}_{15} \mathrm{H}_{19} \mathrm{O}_{5} \mathrm{Br}$ : (359.22): Calcd. C 50.15\%, H 5.33\%; Found: C 50.02\%, H $5.12 \%$.

${ }^{1} \mathrm{H}-\mathrm{NMR}\left(400 \mathrm{MHz}, \mathrm{CDCl}_{3}, \delta /\right.$ p.p.m.): 8.00 (d, $2 \mathrm{H},-\mathrm{CO}$-aromatic), 6.89 (d, $2 \mathrm{H}, \mathrm{O}$ - aromatic), $4.48\left(\mathrm{t}, 2 \mathrm{H},-\mathrm{CH}_{2}-\mathrm{O}\right.$-aromatic), $4.34(\mathrm{q}, 1 \mathrm{H}$, $-\mathrm{CH}-\mathrm{Br}$ ), 4.22 (t, $2 \mathrm{H},-\mathrm{CH}_{2}-\mathrm{O}-\mathrm{CO}-$ ), 4.16 (broad peak, $\left.1 \mathrm{H}, \mathrm{OH}\right), 1.77$ $\left(\mathrm{d}, 1 \mathrm{H},-\mathrm{CH}-\mathrm{CH}_{3}\right)$ and $1.57\left(\mathrm{~s}, 6 \mathrm{H}, \mathrm{CH}_{3}-\mathrm{C}-\mathrm{CH}_{3}\right)$.

${ }^{13} \mathrm{C}-\mathrm{NMR}$ (400 MHz, $\mathrm{CDCl}_{3}$, $\delta /$ p.p.m.): 202.48 ( $C=\mathrm{O}$, ketone), 170.07 $(C=\mathrm{O}$, ester), 162.02, 132.31, 126.39 and 141.11 (aromatic carbons of benzene ring), $75.80(\mathrm{C}-\mathrm{OH}), 65.59\left(\mathrm{CH}_{2} \mathrm{O}\right.$-aromatic $), 63.26\left(\mathrm{CH}_{2} \mathrm{O}-\mathrm{CO}-\right), 39.45$ $(\mathrm{CH}), 28.56\left(\mathrm{CH}_{3}-\mathrm{C}\right)$ and $21.45\left(\mathrm{CH}_{3}-\mathrm{CH}\right)$.
Synthesis of the mid-chain functional macrophotoinitiator Polymerization was performed in a Schlenk tube equipped with a magnetic stirrer. The Schlenk tube was evacuated to a vacuum and then back-filled with nitrogen several times. The catalysts $\mathrm{CuBr}(0.093 \mathrm{~g}, 0.652 \mathrm{mmol})$ and stannous 2-ethylhexanoate $(1.32 \mathrm{mg}, 3.262 \mu \mathrm{mol})$, the ligand $2,2^{\prime}$-bipyridine $(0.305 \mathrm{~g}$, $1.956 \mathrm{mmol})$, the dual-photoinitiator $(\mathrm{Br}-\mathrm{PI}-\mathrm{OH})(0.234 \mathrm{~g}, 0.652 \mathrm{mmol})$ and the monomers $\varepsilon$-caprolactone $(2.89 \mathrm{ml})$ and $\mathrm{St}(3 \mathrm{ml})$ were introduced into the tube under nitrogen atmosphere. The tube was warmed in an oil bath to $110^{\circ} \mathrm{C}$ and stirred for $30 \mathrm{~h}$ at constant temperature. Following polymerization, the mixture was diluted with THF and passed through an alumina column to remove the complex salt. The polymer solution was concentrated and precipitated into 10-fold cold methanol. The polymer (PSt-PI-PCL) was collected after filtration and dried at room temperature in a vacuum. $M_{\mathrm{nGPC}}=8700 \mathrm{~g} \mathrm{~mol}^{-1}$ and $M_{\mathrm{w}} / M_{\mathrm{n}}=1.39$, Conversion $=90 \%$ for St and $81 \%$ for $\varepsilon$-CL.

\section{Photopolymerization}

The photoinitiated free-radical polymerization of the monomer (MMA) in the presence of PSt-PI-PCL was performed in bulk at room temperature. MMA $(1 \mathrm{ml})$ and PSt-PI-PCL $(200 \mathrm{mg})$ were added to a Pyrex tube and degassed with nitrogen prior to irradiation by a 'merry-go-round-type' photoreactor equipped with 16 Philips $8 \mathrm{~W} / 08$ lamps (emitting light nominally at $\lambda>300 \mathrm{~nm}$ ) and a cooling system. After $50 \mathrm{~min}$ of the photopolymerization, the polymer mixture obtained was diluted with $\mathrm{CH}_{2} \mathrm{Cl}_{2}$, precipitated into cold methanol and dried under vacuum at room temperature for $24 \mathrm{~h}$. The conversion was calculated gravimetrically.

\section{Characterization}

${ }^{1} \mathrm{H}-\mathrm{NMR}$ and ${ }^{13} \mathrm{C}-\mathrm{NMR}$ spectra were obtained using an Agilent $400 \mathrm{MHz}$ NMR instrument with $\mathrm{CDCl}_{3}$ as the solvent. Fourier transform infrared (FT-IR) spectra were measured with a Perkin-Elmer Spectrum Two FT-IR spectrophotometer. UV-vis spectra were obtained in a $\mathrm{CH}_{2} \mathrm{Cl}_{2}$ solution with a Shimadzu 1601 spectrophotometer. Fluorescence spectra were registered in a $\mathrm{CH}_{2} \mathrm{Cl}_{2}$ solution with a Jasco FP-6300 spectrofluorometer. The molecular weights and molecular weight distributions were determined by gel permeation chromatography (GPC) using a Viscotek GPCmax Autosampler system consisting of a pump, three Visco-GEL GPC columns (G2000HHR, G3000HHR and G4000HHR), a Viscotek UV detector and a Viscotek differential refractive index detector with a THF flow rate of $1.0 \mathrm{ml} \mathrm{min}^{-1}$ at

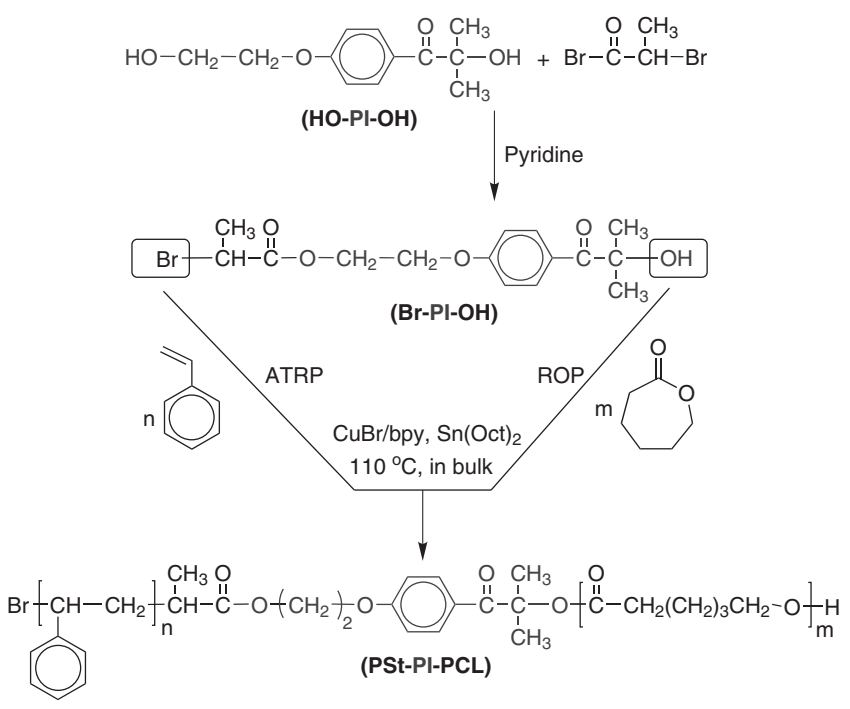

Scheme 1 One-step synthesis of the mid-chain functional macrophotoinitiator of the polystyrene-poly ( $\varepsilon$-caprolactone) diblock copolymer using $\mathrm{Br}-\mathrm{Pl}-\mathrm{OH}$ as the dual-functional photoinitiator. A full color version of this scheme is available at Polymer Journal online. 
$30^{\circ} \mathrm{C}$. GPC was calibrated using PSt standards with a narrow molecular weight distribution. The data were analyzed using Viscotek OmniSEC Omni-01 software.

a<smiles>CC(C)(O)C(=O)c1ccc(OCCOC(=O)C(C)(C)Br)cc1</smiles>

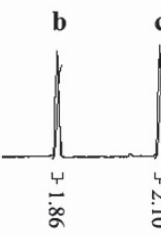

b

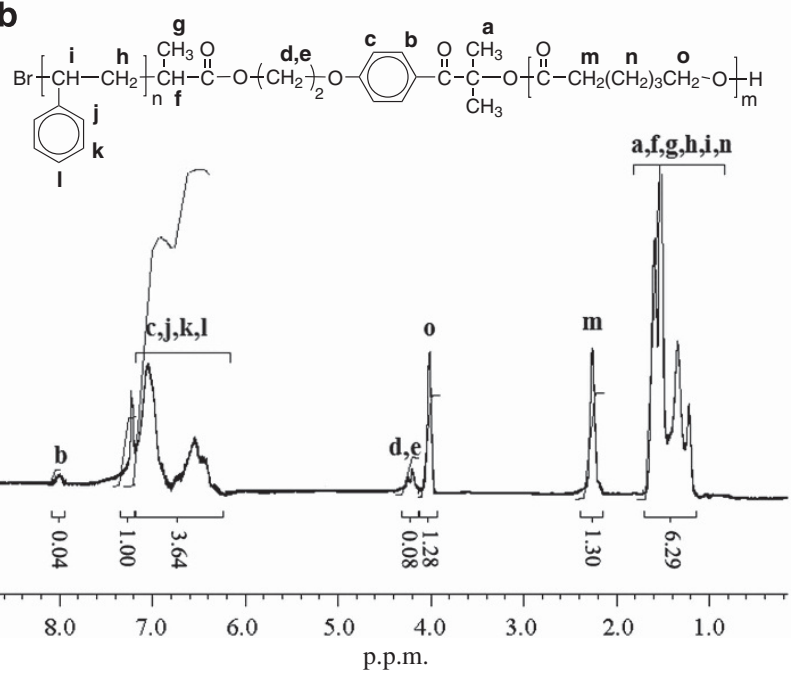

Figure $1{ }^{1} \mathrm{H}$-NMR spectra of the dual-functional photoinitiator Br-PI-OH (a) and the diblock macrophotoinitiator PSt-PI-PCL (b).

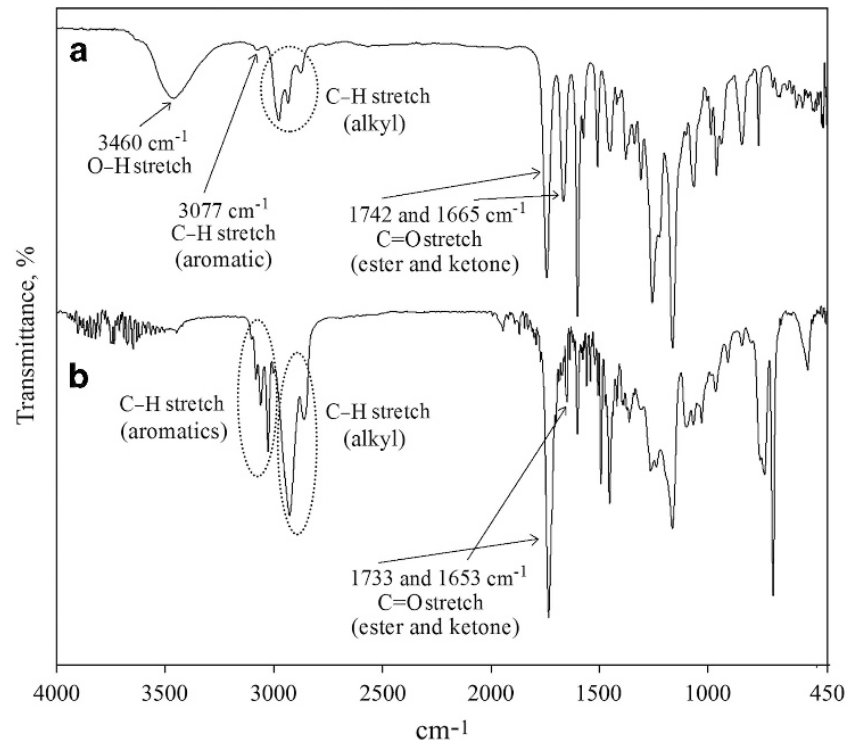

Figure 2 FT-IR spectra of the dual-functional photoinitiator $\mathrm{Br}-\mathrm{PI}-\mathrm{OH}$ (a) and the diblock macrophotoinitiator PSt-PI-PCL (b).

\section{RESULTS AND DISCUSSION}

In the present study, a well-defined mid-chain functional macrophotoinitiator of PSt-PI-PCL was successfully prepared by combining ATRP with ROP in a one-step process using a dual-functional photoinitiator. The dual-functional photoinitiator, Br-PI-OH, was synthesized by a condensation reaction of $\mathrm{HO}-\mathrm{PI}-\mathrm{OH}$ with 2-bromopropanoyl bromide, as shown in Scheme 1 (Reaction 1). The synthesis was performed in an ice-water bath using dry THF as the solvent. The dual-functionalized photoinitiator contains a bromine group that initiates the ATRP of vinyl monomers, such as St, and a hydroxyl functionality, which is the initiation center for the ROP of cyclic lactides or lactones, such as $\varepsilon$-CL.

The successful synthesis of Br-PI-OH was confirmed by characterization with elemental analysis and spectroscopic investigations. The ${ }^{1} \mathrm{H}-\mathrm{NMR}$ spectrum (Figure 1a) exhibited the typical proton signals attributed to aromatic protons at 8.00 and 6.89 p.p.m., a methine

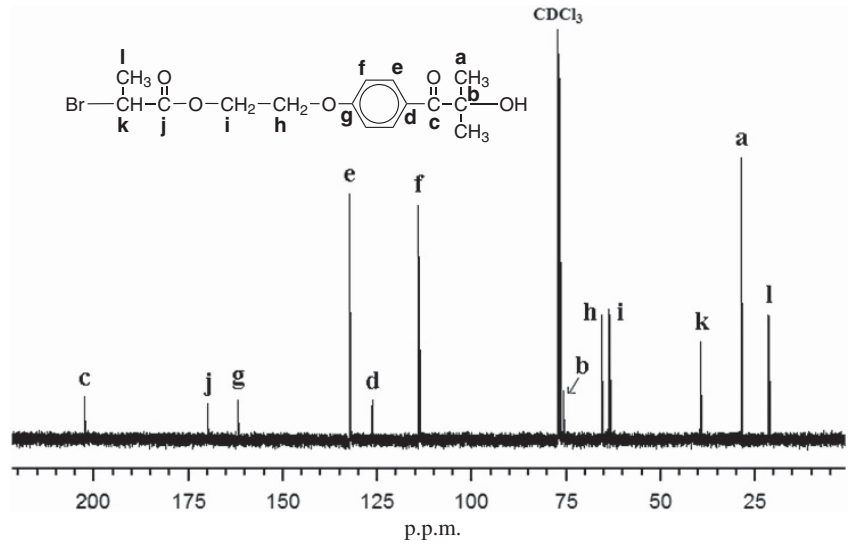

Figure $3{ }^{13} \mathrm{C}-\mathrm{NMR}$ spectrum of the dual-functional photoinitiator $\mathrm{Br}-\mathrm{PI}-\mathrm{OH}$.

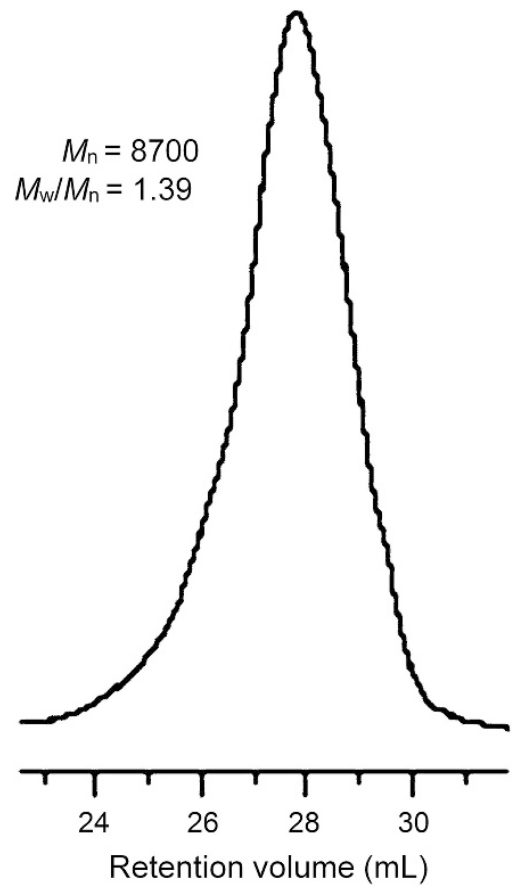

Figure 4 GPC trace of the diblock macrophotoinitiator PSt-PI-PCL. 
$(-\mathrm{CH})$ proton at 4.34 p.p.m., methylene $\left(-\mathrm{CH}_{2}\right)$ protons at 4.48 and 4.22 p.p.m. and methyl $\left(-\mathrm{CH}_{3}\right)$ protons at 1.77 and 1.57 p.p.m. The infrared absorption spectrum (Figure 2a) contained the characteristic $\mathrm{OH}$ alcohol, $\mathrm{C}=\mathrm{O}$ ester and $\mathrm{C}=\mathrm{O}$ keto stretching vibrations at $3460 \mathrm{~cm}^{-1}, 1742 \mathrm{~cm}^{-1}$ and $1665 \mathrm{~cm}^{-1}$, respectively, which also verified the formation of the dual-functional photoinitiator.

The ${ }^{13} \mathrm{C}-\mathrm{NMR}$ spectrum (Figure 3 ) also showed the characteristic carbon peaks expected from Br-PI-OH, which also confirmed the formation of the dual-functional photoinitiator.

The synthesized Br-PI-OH was used as a dual-functional initiator in the one-step polymerization of St and $\varepsilon$-CL monomers to prepare a mid-chain functional macrophotoinitiator of PSt-PI-PCL. During this one-step synthesis, the ATRP of St occurs simultaneously with the ROP of $\varepsilon$-CL to yield the diblock copolymer with a photofunctional group at the mid-point of the polymer chain. The reaction pathway for this process is shown in Scheme 1 (Reaction 2).

The obtained macrophotoinitiator (PSt-PI-PCL) was characterized by ${ }^{1} \mathrm{H}-\mathrm{NMR}$, FT-IR, GPC, UV-vis and fluorescence spectroscopy. In the ${ }^{1} \mathrm{H}-\mathrm{NMR}$ spectrum of PSt-PI-PCL (Figure 1b), along with the

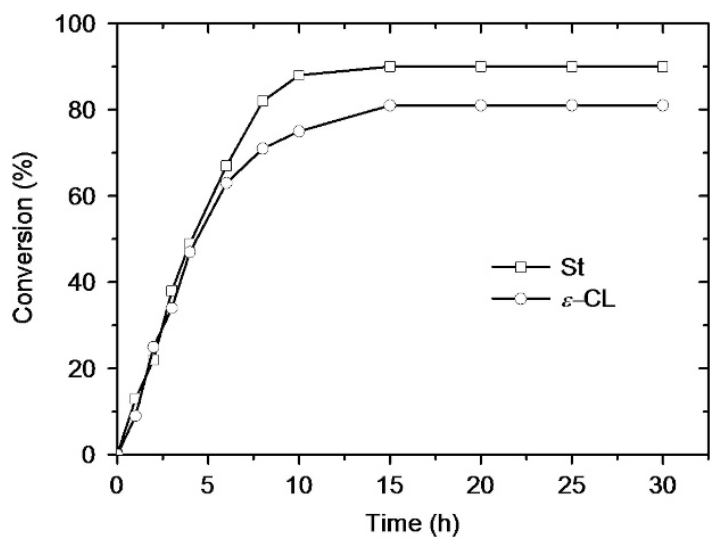

Figure 5 Kinetic study of the one-step polymerization. typical proton signals of PSt and PCL segments, proton signals corresponding to the mid-chain group of the macrophotoinitiator (PI) were observed: the peaks appearing at $4.21-4.17$ p.p.m. were assigned to the PI methylene $\left(-\mathrm{CH}_{2}\right)$ protons. The characteristic aromatic protons of PSt and the methylene $\left(-\mathrm{CH}_{2}\right)$ protons ( $\mathrm{m}$ protons) of the PCL segments were observed at $\sim 7.20-6.25$ and 2.35-2.29 p.p.m., respectively. The related corresponding assignments are given in Figure 1(b). The structure of PSt-PI-PCL was further supported by the FT-IR observations. The FT-IR spectrum contained the characteristic $\mathrm{C}=\mathrm{O}$ ester band of the PCL unit, the aromatic bands of the PSt unit and the $\mathrm{C}=\mathrm{O}$ keto group of the photoinitiator moiety, as shown in Figure 2(b).

The relative molecular weight and the molecular weight distribution of the synthesized PSt-PI-PCL were determined from GPC measurements. As shown in Figure 4, the GPC trace of the PSt-PI-PCL was unimodal and symmetrical $\left(M_{\mathrm{n}}=8700 \mathrm{~g} \mathrm{~mol}^{-1}\right.$ and $\left.M_{\mathrm{w}} / M_{\mathrm{n}}=1.39\right)$. The conversion of the two monomers, the absolute molecular weight and the composition of the block copolymer were determined by the ${ }^{1} \mathrm{H}-\mathrm{NMR}$ measurement shown in Figure 1(b). The conversions of St and $\varepsilon$-CL monomers were calculated as 90 and $81 \%$, respectively, using the characteristic peak intensity of the photoinitiator residue (b protons) located at the junction of the two blocks. The kinetic profile of the one-step polymerization is shown in Figure 5. A decrease in the ATRP rate and an increase in the maximum conversion $(\sim 80 \%)$ of $\varepsilon$-CL were observed. The two polymerization rates were similar until the ROP plateaued at $\sim 80 \%$ conversion of each monomer.

The block copolymer composition was determined as $52.55 \%$ PSt and $47.45 \%$ PCL, in per cent by mole, by comparing the integration ratio of the methylene ( $\mathbf{m}$ protons) peak in the PCL (2.35-2.29 p.p.m.) and the aromatic peaks in the PSt block (7.20-6.25 p.p.m.). The absolute molecular weight $\left(M_{\mathrm{n}, \mathrm{H} \mathrm{NMR}}\right)$ was calculated as $7820 \mathrm{~g} \mathrm{~mol}^{-1}$ $\left(M_{\mathrm{n}, \mathrm{H} \text { NMR }}=7820\right)$ using the following equation (1):

$$
M_{\mathrm{nHNMR}}=\left(I_{\mathrm{m}} / I_{\mathrm{b}}\right) \times M_{\varepsilon-\mathrm{CL}}+\left(2 I_{\mathrm{Ar}} / 5 I_{\mathrm{b}}\right) \times M_{\mathrm{St}}+M_{\mathrm{PI}}
$$

where $M_{\mathrm{n} H \text { NMR }}$ is the ${ }^{1} \mathrm{H}-\mathrm{NMR}$ molecular weight of PSt-PI-PCL; $I_{\mathrm{m}}$, $I_{\mathrm{b}}$ and $I_{\mathrm{Ar}}$ are the integral values of the signals at 2.35-2.29 p.p.m. $\left(\mathrm{CH}_{2}\right.$ protons in the PCL segment), at $8.06-7.98$ p.p.m. (aromatic 2

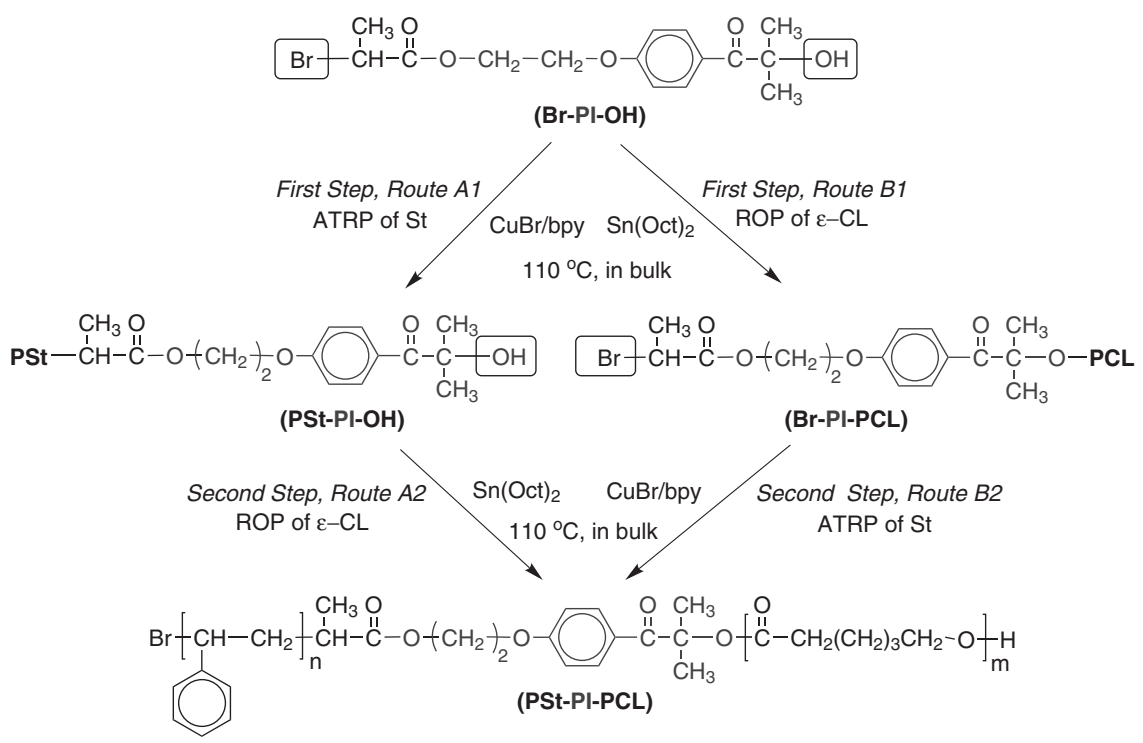

Scheme 2 Two-step synthesis of the mid-chain functional macrophotoinitiator of the polystyrene-poly( $\varepsilon$-caprolactone) diblock copolymer. A full color version of this scheme is available at Polymer Journal online. 
Table 1 Synthesis and properties of the mid-chain functional macrophotoinitiator of the polystyrene-poly( $\varepsilon$-caprolactone) diblock copolymer (PSt-PI-PCL) via a combination of ATRPa and ROPb

\begin{tabular}{|c|c|c|c|c|c|c|c|c|}
\hline Polymer & Initiaor (I) & Monomer & Time (h) & Conv. $^{c}(\%)$ & $M_{n \text { theo }}{ }^{d}$ & $M_{n H N M R^{\mathrm{e}}}$ & $M_{n \cdot G P C^{f}}$ & $M_{\mathrm{w}} / M_{\mathrm{n}}$ \\
\hline \multicolumn{9}{|l|}{ Route A1, A2 } \\
\hline PSt-PI-OH & $\mathrm{Br}-\mathrm{Pl}-\mathrm{OH}$ & St & 7 & 82 & 3775 & 3750 & 3700 & 1.12 \\
\hline PSt-PI-PCL & PSt-PI-OH & $\varepsilon-\mathrm{CL}$ & 35 & 90 & 7884 & 7990 & 7900 & 1.25 \\
\hline \multicolumn{9}{|l|}{ Route B1, B2 } \\
\hline Br-PI-PCL & $\mathrm{Br}-\mathrm{PI}-\mathrm{OH}$ & $\varepsilon-\mathrm{CL}$ & 30 & 93 & 4246 & 4500 & 4400 & 1.43 \\
\hline PSt-PI-PCL & Br-PI-PCL & St & 8 & 75 & 7370 & 7700 & 7500 & 1.35 \\
\hline
\end{tabular}

Abbreviations: ATRP, atom transfer radical polymerization; GPC, gel permeation chromatography; ROP, ring-opening polymerization.

Temperature $=110^{\circ} \mathrm{C},[\mathrm{St}]_{0}=8.75$ the $\mathrm{mol}$ ratio of $[\mathrm{St}]_{0} /[\mathrm{II}] /[\mathrm{CuBr}] /[\mathrm{Bpy}]=40 / 1 / 1 / 3$

bTemperature $=110^{\circ} \mathrm{C},[\varepsilon-\mathrm{CL}]_{0}=9.02 \mathrm{~mol} \mathrm{I}^{-1}$ (in bulk), the mol ratio of $[\varepsilon-\mathrm{CL}]_{0} /[\mathrm{II}]=40 / 1$ and $\left[\mathrm{II} /\left[\mathrm{Sn}(\mathrm{Oct})_{2}\right]=400 / 1\right.$.

${ }^{\mathrm{C}}$ The conversions were calculated as gravimetrically.

${ }^{\mathrm{d}} M_{\mathrm{n} \text { theo }}=[\mathrm{M}]_{0} /\left[\mathrm{II}_{0} \times M_{\text {monomer }} \times\right.$ Conv. $+M_{\text {Initiator }}$

Determined from ${ }^{1} \mathrm{H}$-NMR data.

fDetermined by GPC according PSt standards.

$\mathrm{CH}$ protons of the PI unit) and at 7.20-6.25 p.p.m. (aromatic $\mathrm{CH}$ protons in the PSt segment); $M_{\varepsilon-\mathrm{CL}}, M_{\mathrm{St}}$ and $M_{\mathrm{PI}}$ are the molecular weights of $\varepsilon$-CL, St and Br-PI-OH, respectively. The strong agreement between the $M_{\mathrm{n}}$ data from GPC and the ${ }^{1} \mathrm{H}-\mathrm{NMR}$ measurements suggested that each block copolymer chain contained one photoinitiator group (PI) at the mid-point and that the one-step polymerization reaction proceeded in a controlled manner without mutual interference.

To demonstrate the efficiency of the dual initiator and the controllability of the polymerization reactions, the macrophotoinitiator of PSt-PI-PCL was also synthesized in two steps by a combination of ATRP of St and ROP of $\varepsilon$-CL. As shown in Scheme 2, two routes, ATRP followed by ROP (named route A1 and A2) and ROP followed by ATRP (named route B1 and B2), were attempted to obtain PSt-PIPCL using Br-PI-OH as a dual-functional initiator. The experimental conditions and results are given in Table 1.

${ }^{1} \mathrm{H}-\mathrm{NMR}$ and GPC measurements confirmed the resultant polymer structures. The ${ }^{1} \mathrm{H}-\mathrm{NMR}$ spectra of the samples showed not only specific signals of polymers but also absorptions belonging to the PI moiety. For example, Figure 6 shows the ${ }^{1} \mathrm{H}-\mathrm{NMR}$ spectra of the PSt-PI-OH and Br-PI-PCL precursors in $\mathrm{CDCl}_{3}$.

As shown in Table 1 , the measured and calculated $M_{\mathrm{n}}$ values were in good agreement, indicating that the photoinitiator added to the solution generated macrophotoinitiators with one growing end and one functional end (PSt-PI-OH and Br-PI-PCL), as well as a mid-chain functional macrophotoinitiator of PSt-PI-PCL, with low polydispersities. In all cases, the GPC traces of the polymers were unimodal and narrow, indicating that no side reactions occurred during the polymerization, and well-defined macrophotoinitiators were obtained. These results indicate that both of the routes $(\mathrm{A} 1, \mathrm{~A} 2$ and $\mathrm{B} 1, \mathrm{~B} 2)$ are practical ways of obtaining well-defined PSt-PI-PCL. However, the polydispersities of polymers obtained via route $\mathrm{B} 1, \mathrm{~B} 2$ were slightly broader than those of the polymers obtained via route A1,A2. Therefore, it can be concluded that ATRP followed by ROP (route A1,A2) is the preferred two-step preparation strategy for PSt-PI-PCL with a low molecular weight distribution. The ${ }^{1} \mathrm{H}-\mathrm{NMR}$, UV-vis absorption and fluorescence emission spectra of the PSt-PI-PCL diblock copolymers prepared using the two-step strategies were the same as those synthesized by the one-step method.

UV-vis absorption and fluorescence emission measurements (obtained from the one-step process) also evidenced the formation of PSt-PI-PCL with PI at the mid-chain of the copolymer. The UV-vis spectra of PSt-PI-PCL along with its precursor, Br-PI-OH, in $\mathrm{CH}_{2} \mathrm{Cl}_{2}$
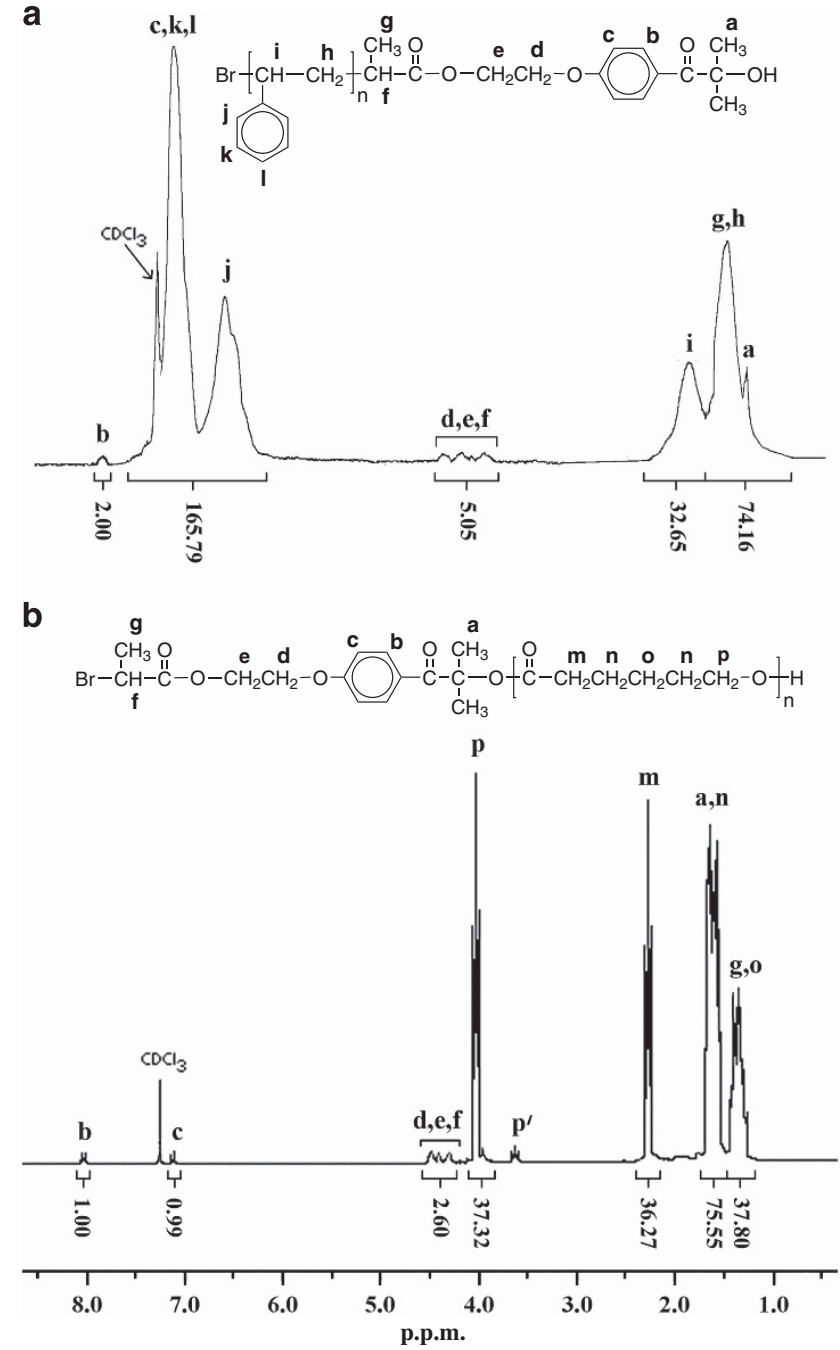

Figure $6{ }^{1} \mathrm{H}-\mathrm{NMR}$ spectra of the macrophotoinitiators PSt-PI-OH (a) and $\mathrm{Br}-\mathrm{PI}-\mathrm{PCL}$ (b).

at room temperature are shown in Figure 7. Each spectrum exhibits the typical absorption band of PI derivatives. The fluorescence emission spectra of PSt-PI-PCL and Br-PI-OH in $\mathrm{CH}_{2} \mathrm{Cl}_{2}$ (Figure 8) show the vibrational structure of the aryl ketone chromophore 


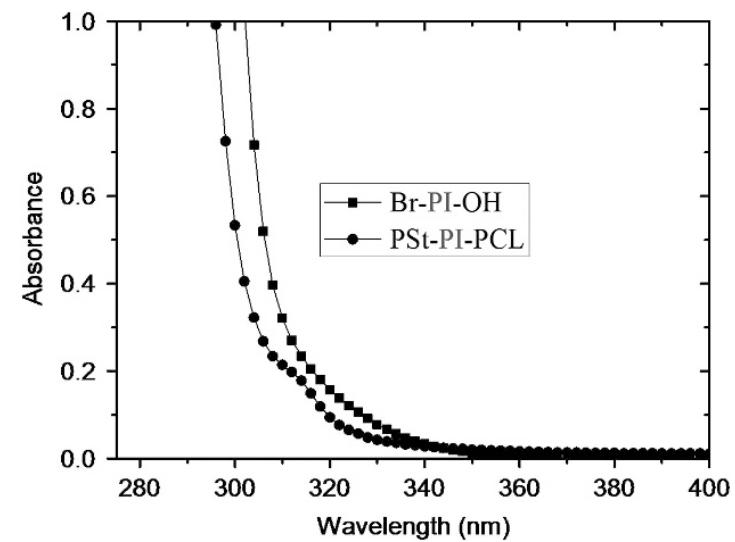

Figure 7 UV-vis absorption spectra of the dual-functional photoinitiator $\mathrm{Br}$ $\mathrm{PI}-\mathrm{OH}\left(6.25 \times 10^{-4} \mathrm{~mol} \mathrm{I}^{-1}\right)$ and the diblock macrophotoinitiator PSt-PI-PCL $\left(7.70 \times 10^{-2} \mathrm{~g} \mathrm{I}^{-1}\right)$ in $\mathrm{CH}_{2} \mathrm{Cl}_{2}$. A full color version of this figure is available at Polymer Journal online.

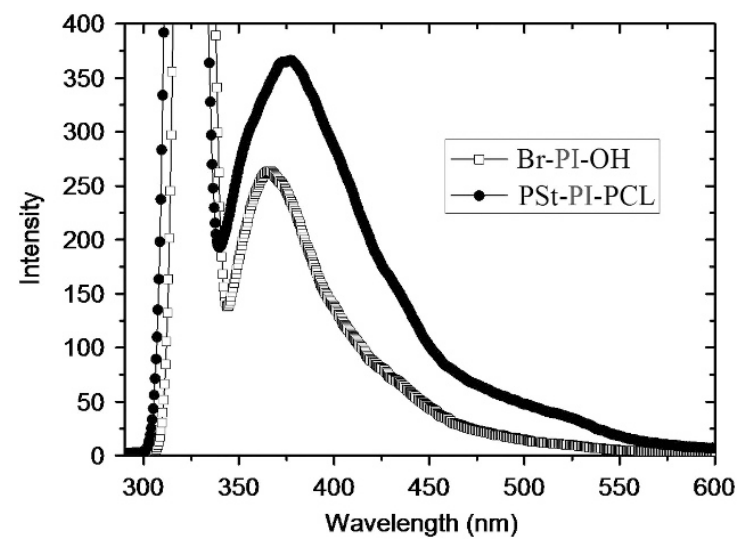

Figure 8 Fluorescence emission spectra of the dual-functional photoinitiator Br-PI-OH $\left(2.50 \times 10^{-4} \mathrm{moll}^{-1}\right)$ and the diblock macrophotoinitiator PSt-PIPCL $\left(4.56 \times 10^{-3} \mathrm{~g} \mathrm{I}^{-1}\right)$ in $\mathrm{CH}_{2} \mathrm{Cl}_{2}, \lambda_{\text {exc }}=325 \mathrm{~nm}$. A full color version of this figure is available at Polymer Journal online.

available in the compounds. The spectroscopic results verify that the photochromophoric PI group was conserved during the one-step polymerization reaction.

The availability of the PI group in the middle of the copolymer chain was further shown via photopolymerization studies of the PSt-PI-PCL macrophotoinitiator produced using the one-step polymerization reaction. The photoinitiated free radical polymerization of MMA with the PSt-PI-PCL macrophotoinitiator in bulk at room temperature produced a $22 \%$ conversion of MMA after $50 \mathrm{~min}$ of irradiation time. A control experiment without the macrophotoinitiator gave only a negligible amount of polymer after the same irradiation time. UV irradiation of the PSt-PI-PCL polymeric photoinitiator caused $\alpha$-scission and yielded two polymer-bound radicals according to the reaction shown in Scheme 3. Both of these radicals may then initiate the polymerization of MMA. As a result, PSt-PMMA and PCL-PMMA block copolymers were obtained. It should be noted that homopolymers of PSt, PCL and poly(methyl methacrylate) might also be present in the product to some extent because of the uncontrollability of the photopolymerization method used in this

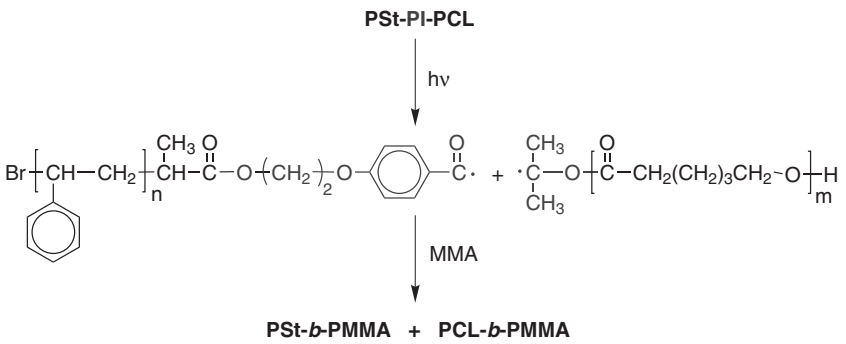

Scheme 3 Photoinitiated free radical polymerization of the diblock macrophotoinitiator PSt-PI-PCL. A full color version of this scheme is available at Polymer Journal online.

study. Thus, the product obtained by this method can be used as a compatibilizing agent for PSt and poly(methyl methacrylate).

In conclusion, a new, well-defined mid-chain functional macrophotoinitiator of PSt-PI-PCL was successfully synthesized in a onestep process using a dual-functional photoinitiator able to combine the ATRP of St with the ROP of $\varepsilon$-caprolactone. The dual-functional photoinitiator was also used as the initiator in a two-step polymerization by sequentially combining ATRP and ROP to obtain the diblock copolymer (PSt-PI-PCL). The polymers produced in both steps have low molecular weight distributions. The structure of the PSt-PI-PCL and the presence of a photochromophoric group (PI) at its mid-point were shown by spectral analyses. The obtained macrophotoinitiator can be used in the photopolymerization of vinyl monomers, such as MMA.

\section{CONFLICT OF INTEREST}

The authors declare no conflict of interest.

\section{ACKNOWLEDGEMENTS}

The authors would like to thank the Harran University, Scientific Research Council (HÜBAK) for its financial support (Project no: 12157).

1 Bernaerts, Katrien V. \& Du Prez, Filip E. Dual/heterofunctional initiators for the combination of mechanistically distinct polymerization techniques. Prog. Polym. Sci. 31, 671-722 (2006).

2 Wang, W. W., Ren, W. Y., Jiang, L. \& Dan, Y. Synthesis and characterization of AB-type copolymers poly(L-lactide)-block-poly(methyl methacrylate) via a convenient route combining ROP and ATRP from a dual Initiator. J. Appl. Polym. Sci. 118, 2379-2388 (2010).

3 Mao, J., Ji, X. \& Bo, S. Synthesis and pH/temperature-responsive behavior of PLLA-bPDMAEMA block polyelectrolytes prepared via ROP and ATRP. Macromol. Chem. Phys. 212, 744-752 (2011).

4 Nasser-Eddine, M., Delaite, C., Hurtrez, G. \& Dumas, P. Controlled one-step synthesis of a diblock copolymer. Eur. Polym. J. 41, 313-318 (2005).

5 Shin, S. J., Yu, Y. C., Seo, J. D., Cho, S. J. \& Youk, J. H. One-Step Synthesis of poly(Nvinylpyrrolidone)-b-poly(L-lactide) block copolymers using a dual Initiator for RAFT polymerization and ROP. J. Polym. Sci. Part A: Poly. Chem. 52, 1607-1613 (2014).

6 Mecerreyes, D., Moineau, G., Dubois, P., Jerome, R., Hedrick, J. L., Hawker, C. J., Malmström, E. E. \& Trollsas, M. Simultaneous dual living polymerizations: A novel onestep approach to block and graft copolymers. Angew. Chem. Int. Ed. 37, 1274-1276 (1998).

7 Hawker, C. J., Hedrick, J. L., Malmström, E. E., Trollsas, M., Mecerreyes, D., Moineau, G., Dubois, P. \& Jerome, R. Dual living free radical and ring opening polymerizations from a double-headed initiator. Macromolecules 31, 213-219 (1998).

8 Öztürk, T., Göktaş, M. \& Hazer, B. One-step synthesis of triarm block copolymers via simultaneous reversible-addition fragmentation chain transfer and ring-opening polymerization. J. App. Polym. Sci. 117, 1638-1645 (2010).

9 Kang, H. U., Yu, Y. C., Shin, S. J. \& Youk, J. H. One-step synthesis of block copolymers using a hydroxyl-functionalized trithiocarbonate RAFT agent as a dual initiator for RAFT polymerization and ROP. J. Polym. Sci. Part A: Poly. Chem. 51, 774-779 (2013). 
10 Yu, Y. C., Li, G., Kang, H. U. \& Youk, J. H. One-step synthesis of poly(alkyl methacrylate)-b-polyester block copolymers via a dual initiator route combining RAFT polymerization and ROP. Colloid. Polym. Sci. 290, 1707-1712 (2012).

11 Kryuchkov, M. A., Detrembleur, C. \& Bazuin, C. G. Linear amphiphilic diblock copolymers of lactide and 2-dimethylaminoethyl methacrylate using bifunctionalinitiator and one-pot approaches. Polymer 55, 2316-2324 (2014).

12 Corrales, T., Catalina, F., Peinado, C. \& Allen, N. S. Free radical macrophotoinitiators: an overview on recent advances. J. Photochem. Photobiol. A Chem. 159, 103-114 (2003).

13 Yagci, Y., Mishra, M. K. Macroinitiators, in: Macromolecular Design: Concept and Practice (ed. Mishra M. K.) Ch. 6, 229-264 (Polymer Frontiers International, inc., New York, NY, USA 1994).

14 Yagci, Y. Photoinitiating systems and their use in polymer synthesis. Macromol. Symp. 161, 19-36 (2000).

15 Wang, Y. L., Jiang, X. S. \& Yin, J. A. Water-soluble supramolecular-structured photoinitiator between methylated b-cyclodextrin and 2,2-dimethoxy-2-phenylacetophenone. J. Appl. Polym. Sci. 105, 3819-3823 (2007).

16 Yagci, Y., Jockush, S. \& Turro, N. J. Photoinitiated polymerization: advances, challenges, and opportunities. Macromolecules 43, 6245-6260 (2010).

17 Fouassier, J. P. \& Lalevee, J. Photoinitiators for Polymer Synthesis: Scope, Reactivity and Efficiency (Wiley-VCH Verlag GmbH \& Co., KGaA, Weinheim, Germany, 2012).

18 Durmaz, Y. Y., Karagoz, B., Bicak, N. \& Yagci, Y. Synthesis of block copolymers by combination of ATRP and photoiniferter processes. Polym. Int. 57, 1182-1187 (2008).

19 Durmaz, Y. Y., Tasdelen, M. A., Aydogan, B., Kahveci, M. U., Yagci, Y. Light Induced Processes for the Synthesis of Polymers with Complex Structures, The NATO Science For Peace and Security Programme (eds Khosravi E., Yagci Y. \& Savelyev Y.) Part 5, 329-341 (Springer, Dordrecht, the Netherlands, 2009).

20 Degirmenci, M., Sarac, M. A. \& Genli, N. Synthesis and characterization of mid-chain macrophotoinitiator of poly ( $\varepsilon$-caprolactone) by combination of ROP and click chemistry. Polym. Bull. 71, 1743-1755 (2014).
21 Degirmenci, M., Cianga, I., Hizal, G. \& Yagci, Y. Synthesis, characterization and application of polymeric photoinitiators prepared by atom transfer radical polymerization and ring-opening polymerization. Poly. Preprints 43, 22-23 (2002).

22 Degirmenci, M. \& Genli, N. Synthesis of well-defined telechelic macrophotoinitiator of polystyrene by combination of ATRP and click chemistry. Macromol. Chem. Phys. 210, 1617-1623 (2009).

23 Temel, G. \& Arsu, N. One-pot synthesis of water-soluble polymeric photoinitiator via thioxanthonation and sulfonation process. J. Photochem. Photobiol. A: Chem. 202, 63-66 (2009).

24 Durmaz, Y. Y., Yilmaz, G. \& Yagci, Y. N-alkoxy pyridinium ion terminated polystyrenes: a facile route to photoinduced block copolymerization. J. Poly.Sci.: Part A: Poly. Chem. 45, 423-428 (2007).

25 Degirmenci, M., Cianga, I. \& Yagci, Y. Synthesis of well-defined polystyrene macrophotoinitiators by atom-transfer radical polymerization. Macromol. Chem. Phys. 203, 1279-1284 (2002).

26 Degirmenci, M., Alter, S. \& Genli, N. Synthesis and characterization of well-defined mid-chain functional macrophotoinitiators of polystyrene by combination of ATRP and "click" chemistry. Macromol. Chem. Phys. 212, 1575-1581 (2011).

27 Degirmenci, M. Synthesis and characterization of novel well-defined end-functional macrophotoinitiator of poly(MMA) by ATRP. J. Macromol. Sci. Part A—Pure Appl. Chem. 42, 21-30 (2005).

28 Degirmenci, M., Hizal, G. \& Yagci, Y. Synthesis and characterization of macrophotoinitiators of poly ( $\varepsilon$-caprolactone $)$ and their use in block copolymerization. Macromolecules 35, 8265-8270 (2002).

29 Degirmenci, M. Synthesis of novel well-defined end-functional macrophotoinitiator of $\operatorname{poly}(\varepsilon$-caprolactone) by ring-opening polymerization. Polym. J. 36, 542-548 (2004).

30 Degirmenci, M., Besli, P. A. \& Genli, N. Synthesis of a well-defined end-chain macrophotoinitiator of poly $(\varepsilon-$ caprolactone $)$ by combination of ring-opening polymerization and click chemistry. J. Polym. Res. 21, 540-549 (2014). 